\title{
Mediator-Assisted Multi-Source Routing in Information-Centric Networks
}

\author{
Vassilios G. Vassilakis*, Laura Carrea ${ }^{\dagger \ddagger}$, Ioannis D. Moscholios ${ }^{\S}$, Michael D. Logothetis \\ * School of Computing \& Engineering, University of West London, London, United Kingdom \\ $\dagger$ School of Computer Science \& Electronic Engineering, University of Essex, Colchester, United Kingdom \\ $¥$ School of Mathematical \& Physical Sciences, University of Reading, Reading, United Kingdom \\ $\S$ Dept. of Informatics \& Telecommunications, University of Peloponnese,Tripolis, Greece \\ I Dept. of Electrical \& Computer Engineering, University of Patras, Patras, Greece
}

\begin{abstract}
Among the new communication paradigms recently proposed, information-centric networking (ICN) is able to natively support content awareness at the network layer shifting the focus from hosts (as in traditional IP networks) to information objects. In this paper, we exploit the intrinsic content-awareness ICN features to design a novel multi-source routing mechanism. It involves a new network entity, the ICN mediator, responsible for locating and delivering the requested information objects that are chunked and stored at different locations. Our approach imposes very limited signalling overhead, especially for large chunk sizes (MBytes). Simulations show significant latency reduction compared to traditional routing approaches.
\end{abstract}

Keywords-information-centric networking; multi-source routing; named-data networking.

\section{INTRODUCTION}

In the recent years, we are observing new communication paradigms coming into the scene and many established concepts are being revisited. One such new paradigm is the information-centric networking (ICN) concept which has been attracting increasing attention of the research community who attempts to apply its concepts into a wide range of communication networks, such as the Internet, mobile networks, and satellite networks.

The appearance of ICN has been motivated by the intrinsic inefficiencies of the current host-to-host communication paradigm which is used to deliver content. In fact, the Internet today is mainly used for delivering multimedia content to end users, rather than for connecting hosts. The user is interested in the content itself, while the location of the content is usually of minor importance or totally irrelevant. However, the current Internet architecture, that has been designed several decades ago, is mainly based on the host-centric communication paradigm. As numerous research studies indicate, many of the current Internet's limitations in the areas of effective content dissemination and efficient use of processing and storage resources, lie in its host-centric nature (e.g., [1], [2]).

For example, the fast proliferation of mobile Internet and mobile social networks has led to the situation where many popular information objects (e.g., pictures and video clips) have been replicated in multiple locations (sources), but at the same time no appropriate mechanisms have been developed for locating the best (e.g., closest) replica to the interested user. Hence, the development of fast and efficient multi-source routing mechanisms is of major importance.
Motivated by the aforementioned limitations of the current Internet, the concept of ICN has emerged. ICN is a novel technology that provides access to named information objects as a native network service, aiming at higher efficiency and better security than today's host-based communication mechanisms. ICN aims at shifting the focus from the hosts to the information objects as well as to provide new and enhanced services to fixed and mobile users. By promoting the information objects to the first class citizens, ICN is able to natively support content awareness at the network layer. This is different from the IP layer of traditional networks, which is content-agnostic. According to the traditional host-centric approach, users send requests containing the IP address of the remote host (e.g., a web server) that stores the desired information object (e.g., a web page). On the other hand, according to the ICN approach, users request a named information object, specified, e.g., in the form of a unique name or identifier of the object. After the request has been issued, it is then the duty of the network to find the most appropriate location to retrieve the object from. This essentially means, that to cope with efficient information dissemination the network needs to become more intelligent by understanding the delivered content, rather than being used simply as a bit pipe. This network intelligence can be exploited in a number of ways to enable multi-source content delivery.

Some early ICN developments have been done by a number of research initiatives, including DONA [3], NDN/CCN [4], SAIL [5], COMET [6], and PURSUIT/PSIRP [7]. Several good surveys on ICN have been published (e.g., [8] - [10]). Furthermore, some recently started research projects apply the ICN concepts in other domains, such as delay-tolerant networks [11] and community mesh networks [12], [13].

Despite the variety of proposed ICN approaches, they share one common characteristic. Each information object (e.g., a movie file) has a unique name bound to it (and there are typically mechanisms to validate and secure this binding). This feature enables the use of in-network storing of information to speed up the content distribution [14], [15]. The in-network storage of information is a concept that is already known and used in the current Internet, the well-known caching operation (e.g., Web caching and peer-to-peer caching). However, the absence of a unified naming system for identical information objects makes it inefficient, especially when considering multisource content delivery. For example, two copies of the same object held at different servers will be treated as two different objects by the Web caching system. 
In this paper, we exploit the intrinsic content-awareness ICN features for the design of a novel ICN architecture based on a multi-source routing mechanism. The proposed architecture has been inspired by the named data networking (NDN) architecture ([4]) but it introduces a mediator entity which is responsible for enabling and managing the multisource routing mechanism. As recently pointed out, multisource multicast routing is not natively scalable in NDN [16].

Our proposed routing scheme exploits the unique naming capabilities of ICN to enable a more efficient content distribution. While the locations of the objects are identified, very little signalling or computational overhead is introduced for notifying the appropriate sources and configuring the in-path routers.

This paper is organized as follows. In Section II, we present the related work on multi-source routing. In Section III, the ICN architecture proposed in this paper is introduced. In Section III, we also describe our proposed mediator-assisted multi-source routing mechanism and in Section IV, we present our simulation results. We compare the proposed approach with the traditional one in terms of efficiency and content access response times. We conclude and discuss our future work in Section V.

\section{RELATED WORK}

The concept of ICN redefines the way to do networking so that many established networks fundamentals are being revisited, especially in the areas of network efficiency and traffic engineering [17], [18], network coding [19], [20], satellite communications [21], [22], network security [23], [24] and mobility support [25].

Regarding routing in ICN, a recent survey [26] describes the efforts in the research community towards content naming and content-based routing for different ICN architectures, both clean slate and incremental. The routing mechanism choice is dependent on the naming of the information content and on the primitives of the architecture. For the Interestbased NDN/CCN architecture, the communication primitives are expressing-an-interest and requesting-data. Information is identified through names and consist of a human-friendly part and a secure hash algorithm 256 (SHA-256) digest of the content. In this way, information naming is unique. A client broadcasts the interest for a content over all available connections. Any NDN node having the original or replicated copy of that content or caching it, is able to reply to the message with the corresponding data. The content and its request are exchanged using the content name. The NDN nodes maintain the tables to store probable source(s) for data (forwarding information base, FIB), the return path state for possible data messages in response to the interests forwarded upstream (pending interest table, PIT) and tables for caching Data (content store, CS). Routing is performed depending on the component structure of names. Prefix aggregation and loop free forwarding are performed to achieve routing table compression and reduced messaging over head. The routing protocol is based on open shortest path first (OSPF) adapted for named data and it populates the routing tables.

Another clean slate ICN architecture is the PURSUIT/PSIRP where the content is labeled with randomly looking identifiers (hierarchically organized) and the communication primitives are publish and subscribe. The publications are matched to the subscriptions by the rendezvous function while the actual delivery tree from the publisher(s) to the subscriber(s) is built by the topology management function, which is centralized [27]. However, the routing mechanism being source routing-based suffers from the security problems inherent in source routing mechanisms [28], [29].

Routing from multiple sources has been proposed in peerto-peer networks [30], wireless sensor networks [31], wireless networks [32], and recently in content centric networks [20] but never at the lower layers of the network architecture. Only very recently, multisource routing has been attempted in ICN [16] within the NDN/CCN architecture where multisource multicasting can not be natively supported due to scaling limitations. In fact, to do so each multicast source must be known in the network and a tree to each such source needs to be maintained. The core of the multi-source multicast routing lies in a multi-instantiated destination spanning tree (MIDST) for each name prefix that denotes a multicast group. The MIDST connects all the anchors of a multicast group which are routers that advertise having an instance of a multicast group locally available. Routing to the nearest sources of the multicast group can be provided by the routers using a content routing protocol like NLSR [33] or DCR [34] while the information centric multicast (ICM) entity builds and maintains the MIDST of the multicast group using the nearestinstance routing information provided by the routing protocol.

The architecture proposed in this paper is a variation of the NDN/CCN architecture and it is described in details in the following section. The main difference lies in the ICN mediator entity which is responsible for appropriately configuring content routers to ensure content delivery from publisher(s) to subscriber(s).

\section{INFORMATION-CENTRIC NETWORK MODEL}

In this section, we describe our proposed model of an ICN. Initially we introduce the network architecture and network elements. Then, we describe the basic network functionality, such as issuing publication and subscription messages. Finally, we describe a more advanced functionality, such as mediatorassisted multi-source content routing.

\section{A. Basic Functionality: Publications and Subscriptions}

As mentioned in Section I, in an ICN, each information object is distinguished by unique object identifier (O-ID). If the object is large (e.g., a large movie clip), it can be considered consisting of a finite number of chunks. For example a 5GB file may consist of $1 \mathrm{~K}$ chunks of $5 \mathrm{MB}$ each. Similarly to objects, each chunk is also distinguished by a unique (within the object) chunk identifier (C-ID). Therefore, the pair $<\mathrm{O}$ ID,C-ID $>$ can be used as a unique identifier of a chunk in the network. A chunk, as we will see below, is the smallest routable and storable quantity in an $\mathrm{ICN}^{1}$.

Note that the problem of object naming (i.e., designing appropriate structures for unique object names or IDs) is outside

\footnotetext{
${ }^{1}$ In the same way that an IP packet is the smallest routable quantity at the network layer of traditional host-centric networks
} 


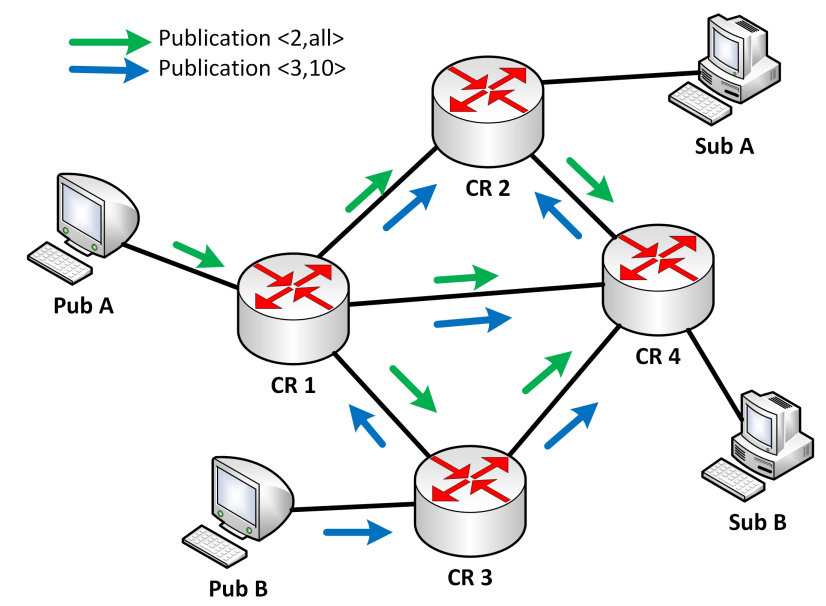

Fig. 1. An example of issuing publication messages in a small informationcentric network with two publishers, two subscribers, and four content routers.

the scope of this paper. This problem has been studied in the literature (e.g., [35]) and a number of alternative solutions have been proposed.

To illustrate the basic ICN functionality of issuing publication messages, consider the small information-centric network depicted in Fig. 1. We distinguish three different network elements: a) publishers (Pub), b) subscribers (Sub), and c) content routers $(\mathrm{CR})$.

1) Issuing publication messages:

Publishers are those who possess information objects ${ }^{2}$ that they wish to share with a set of subscribers.

Publishers advertise the availability of the content objects by issuing the publication messages in the network. A typical way of disseminating the publication messages is flooding. A content router upon receiving a publication message, records a state (O-ID, C-ID, and incoming interface), in order to serve any future requests for this object. The state is kept in the routing table.

The contents of the routing tables of the simple example of Fig. 1 are presented in Fig. 2. These are the table contents upon receiving the publication messages sent by Pub A and Pub B. We observe that Pub A advertises all chunks of object 2 , whereas Pub B advertises the 10th chunk of object 3 . The interface from where the publication message was received is recorded in the "next hop" 3 field of the table.

Note that, the next hop field of the routing table may contain multiple entries. This essentially means, that some chunks can be reached via multiple paths. Hence, the CR may request chunks via a random next hop or select the next hop in round robin fashion. Also, note that not all paths have the same length. For example, the first entry in the routing table of CR 4 indicates that the object 2 can be reached via CR 1, CR2, and CR3. As can be observed in Fig. 1, getting the object via CR 2 would be faster (i.e., only 2 hops). However, CR 4 does not have the knowledge of path lengths, since CRs

\footnotetext{
${ }^{2}$ We also allow for the publishers to posses a part of an object (i.e., a subset of the object chunks)

${ }^{3}$ This field is named "next hop" because this will be the interface(-s) where the corresponding subscription messages will be forwarded.
}

\begin{tabular}{|c|c|c|c|c|c|}
\hline \multicolumn{3}{|c|}{ Routing Table at CR 1 } & \multicolumn{3}{c|}{ Routing Table at CR 2 } \\
\hline Object ID & Chunk ID & Next hop & Object ID & Chunk ID & Next hop \\
\hline 2 & all & Pub A & 2 & all & CR 1 \\
\hline 3 & 10 & CR 3 & 3 & 10 & CR 1, CR 4 \\
\hline Routing Table at CR 3 & & Routing Table at CR 4 \\
\hline Object ID & Chunk ID & Next hop & Object ID & Chunk ID & Next hop \\
\hline 2 & all & CR 1 & 2 & all & CR 1, CR 2, \\
\hline 3 & 10 & Pub B & 3 & 10 & CR 1, CR 3 \\
\hline 3
\end{tabular}

Fig. 2. Routing tables of four content routers of the simple informationcentric network example.

do not have the global view of the network topology. An easy solution to this would be to add additional field indicating the distance to the chunk for each possible next hop or other useful contextual information to select the best path. This problem has been studied in [36] and will not be investigated in our work.

2) Issuing subscription messages:

Subscribers are those who are interested in receiving the published information objects. Subscribers issue the subscription messages in the network to indicate their interest in receiving one or more chunks of an object. To illustrate this concept, we continue the example of Figs. 1 and 2. As depicted in Fig. 3, Sub A sends the subscription message (orange arrow), for the 10th chunk of object 3 , to its attached router CR 2. The latter, having previously received a publication message from Pub B through CR 1 and CR 4 (as indicated in the routing table), will forward the subscription message to $\mathrm{CR} 4^{4}$. At the same time, CR 2 will add a corresponding record in the pending interest table (PIT), as shown in Fig. 4. This recorded state will be later used by CR 2 to decide where (i.e., to Sub A in this case) to forward the received data message containing the requested object. Next, CR 4 will forward the subscription

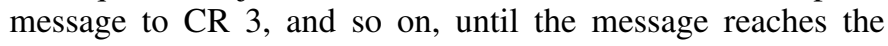
entity (i.e., Pub B) that has advertised the particular object. Finally, Pub B will send the requested object, which will be delivered to Sub B following the reverse path according to the corresponding entries in PITs (Fig. 4).

In the literature, two different subscription approaches have been considered: a) file-level and b) chunk-level. In the former, a single subscription message is used to request the whole file, while in the latter, each chunk requires a dedicated subscription massage. There are pros and cons to both approaches. For example, the file-level subscription approach introduces less traffic in the network, but suffers from larger delays in case of lost subscription messages. In our work, as can be seen in the above examples, we adopt a hybrid approach. That is, the subscriber is able to request (using a single message) either the whole file, or a single chunk, or multiple chunks.

\section{B. Support for Multi-Source Content Routing}

The basic ICN functionality, described in the previous subsection, enables only a single publisher to respond to

\footnotetext{
${ }^{4}$ Assume that CR 2 for some reason selected CR 4 over CR 1 in this particular case.
} 


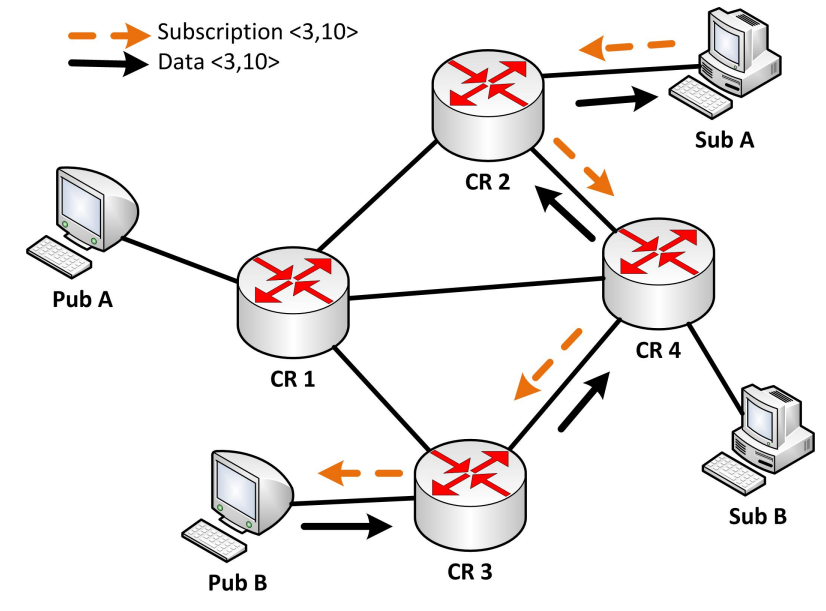

Fig. 3. An example of issuing subscription messages and sending data packets in a small information-centric network with two publishers, two subscribers, and four content routers.

\begin{tabular}{|c|c|c|c|c|c|}
\hline \multicolumn{3}{|c|}{ Pending Interest Table at CR 2 } & \multicolumn{3}{|c|}{ Pending Interest Table at CR 4 } \\
\hline Object ID & Chunk ID & $\begin{array}{c}\text { Next hop } \\
\text { (to Sub) }\end{array}$ & Object ID & Chunk ID & $\begin{array}{c}\text { Next hop } \\
\text { (to Sub) }\end{array}$ \\
\hline 5 & $1,2,3$ & CR 4 & 8 & $11-20$ & Sub B \\
\hline 3 & 10 & Sub A & 3 & 10 & CR 2 \\
\hline & Pending Interest Table at CR 3 \\
\hline
\end{tabular}

Fig. 4. Pending interest tables of three content routers of the simple information-centric network example.

each subscription message. One of the reasons for that is the fact that publication messages do not carry any information about the publisher (of a particular object). Hence, a CR knows the publisher only if the latter is CR's neighbour. For example, in Fig. 1, CR 4 does not know that the publisher of $<3,10>$ is Pub B. Furthermore, CR 4 does not even know the number of publishers (of a particular object). That is, the two publication messages that CR 4 receives from CR 1 and CR 3 could correspond to two different publishers (e.g., Pub A and Pub B) or to a single publisher (Pub B in this example). Hence, the mechanism described above does not support multisource routing (i.e., retrieving (parts of) the requested object from multiple sources (publishers)). However, this feature is often desirable for load balancing, latency reduction, and even security reasons.

Below, we introduce appropriate modification to the basic ICN model and the communication protocol, in order to support multi-source content routing. To this end we adopt a mediation-based approach. According to it, a new (logical) entity, which we call mediator (Med), is responsible for receiving the publication and subscription messages and for appropriately configuring CRs to ensure content delivery from publisher(s) to subscriber(s). This concept is illustrated in Fig. 5. In this example, Pub A and Pub B send the publication messages to Med. In the general case, the published objects could be different, but for the sake of a multi-source example

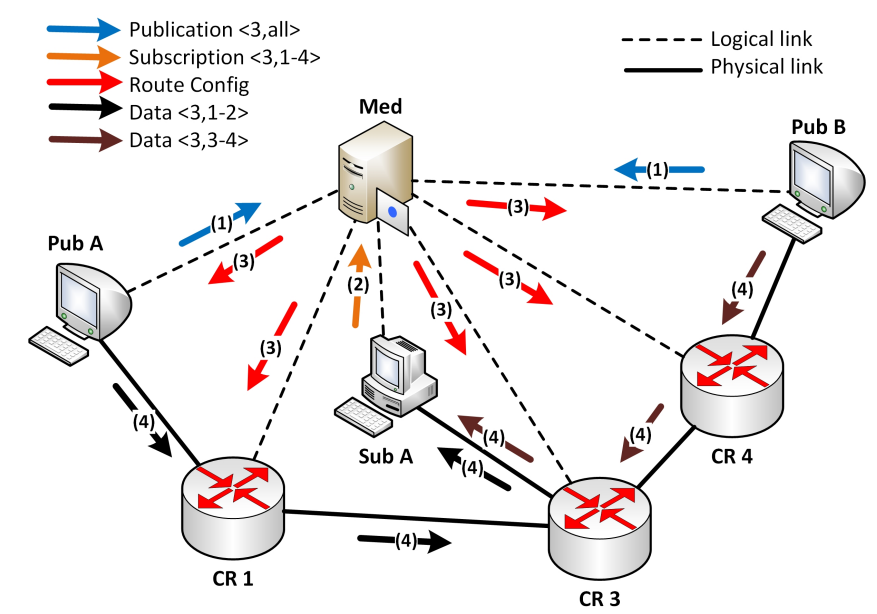

Fig. 5. Illustrating the mediator-assisted multi-source content routing in a small information-centric network with two publishers, one subscriber, three content routers, and one mediator.

let us assume that both publishers possess and advertise the same object (e.g., <3,all $>$ ). Sub A subscribes to the $<3,1-$ $4>$ by sending a message to Med. The latter is assumed to be aware of the network topology and can decide that, for example, chunks 1 and 2 will be served by Pub A, whereas chunks 3 and 4, from Pub B. To achieve that, Med will send route configuration messages to all involved Pubs and CRs. In Fig. 5 on the arrows we indicate a typical order of sending the messages. That is, initially publication and subscription messages are received. Then, Med sends route configuration messages and, finally, publishers start sending data. However, our proposed protocol does not enforce a particular order of messages. In some scenarios, for example, route configuration messages might be sent before publication and/or subscription messages are received.

Mediation systems have also been used in other ICN architectures. For example, in the PURSUIT ICN [7] the mediation system consists of the topology management function [38], responsible for constructing the delivery tree between publisher(s) and subscriber(s), and the rendezvous function [39], responsible for matching publisher(s), subscriber(s), and content objects. The mediation system can either determine the shortest path or a path based on predefined traffic engineering objectives [17]. However, differently to our proposed approach, in the PURSUIT architecture the mediation system will send the delivery path information (sequence of CRs) to the publisher, so that the publisher can send the requested object to the subscriber(s). This effectively implements a source routing mechanism. Hence, this approach suffers from the security problems inherent in source routing mechanisms, in both traditional networking [40], [41] and in ICN [28], [29].

\section{EVALUATION}

In this section we compare our proposed multi-source routing mechanism with the traditional ICN routing mechanism. The comparison is performed by means of computer simulations with NS-3 [42]. 


\section{A. Performance Metrics}

We consider real-time applications, such as on-demand video streaming. The popularity of these types of applications has risen significantly in the recent years and they are expected to gain benefits from multi-source routing.

Consider the set, $S$, of subscribers and the set, $F$, of available files in an ICN. We calculate the average response time, $E\left[R T_{s}^{r}(f)\right]$, of a subscriber $s \in S$ when requesting a file $f \in F$ using a routing mechanism $r . R T_{s}^{r}(f)$ is defined as the elapsed time between the beginning of the subscription message and the reception of the first data chunk of the requested file. We also calculate the efficiency of our proposed multi-source routing mechanism $r$, according to the following formula:

$$
E f f(M S)=1-\frac{E\left[R T_{s}^{M S}(f)\right]}{E\left[R T_{s}^{T R}(f)\right]}
$$

where $M S$ and $T R$ refer to the multi-source and traditional routing mechanisms, respectively. The highest value of $E f f(M S)$ is 1 , when $E\left[R T_{s}^{M S}(f)\right] \rightarrow 0$. The lowest value of $\operatorname{Eff}(M S)$ is 0 , when $E\left[R T_{s}^{M S}(f)\right]=E\left[R T_{s}^{T R}(f)\right]$.

\section{B. Experimental Setup}

We consider a random network topology consisting of 100 network elements (NEs). These include 10 subscribers and a variable number of publishers: $10,20, \ldots, 90$. The rest of the NEs are CRs. The average number of hops between two NEs is 5. Each link has a bandwidth of $10 \mathrm{Gbps}$ and a latency of $10 \mathrm{~ms}$. In the first scenario, we consider a variable number of information objects (files), from 100 to $1 \mathrm{~K}$, as shown in the horizontal axis of Fig. 6. Each file has the size of 5GB and is divided into $1 \mathrm{~K}$ chunks of $5 \mathrm{MB}$. These files are placed randomly in the network at the publishers. We consider file requests originated at random places in the network.

\section{Simulation Results}

In Fig. 6, we present the simulation results for the response time achieved by both routing mechanisms. For the case of the traditional routing mechanism, we also present results for a varying number of publishers (10, 30, and 50). We observe, that in all cases the MS mechanism outperforms the TR mechanism. We also observe that both mechanism perform better when the number of requests is smaller. Finally, although the TR mechanism achieves smaller response time when the number of publishers is increasing, it still remains higher than what is achieved by the MS mechanism.

In Fig. 7, we present the efficiency $\operatorname{Eff}(M S)$ of the MS mechanism for varying number of files and publishers. We observe that the efficiency is over $50 \%$ when the number of publishers is 10 and is decreasing as the number of publishers increases.

\section{CONCLUSion And Future Work}

In this paper we presented a mediator-assisted multi-source routing mechanism for ICN. The proposed approach exploits the content-awareness feature of ICN at the network layer. The introduced processing and signalling overhead is relatively small. On the other hand, as indicated by simulation results,

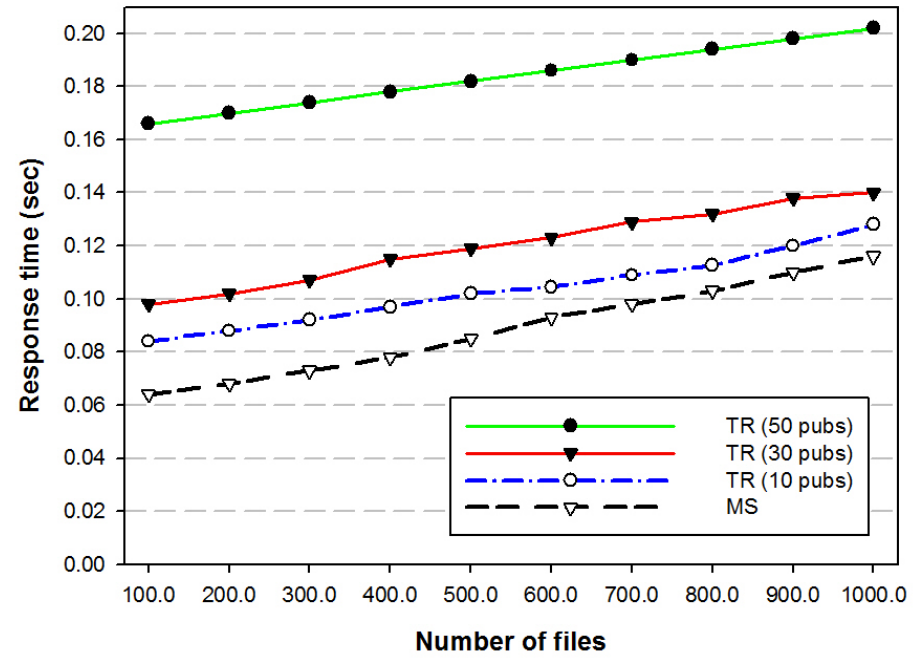

Fig. 6. Simulation results of the response time vs the number of files for the multi-source and the traditional routing mechanisms in an information-centric network.

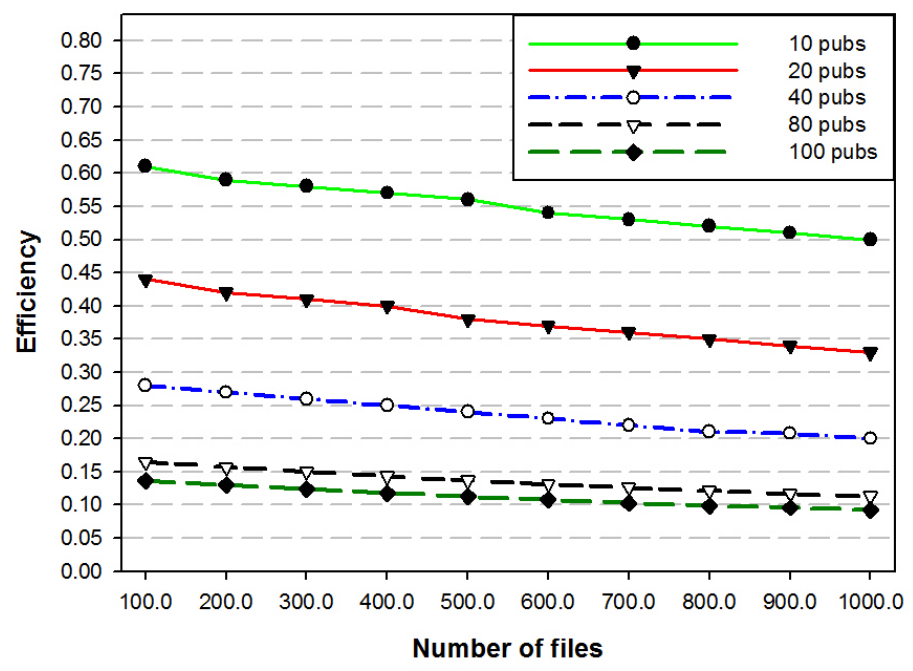

Fig. 7. Simulation results for the efficiency of multi-source routing vs the number of files for a varying number of publishers in an information-centric network.

significant performance improvements can be achieved. Simulation results show an up to $50 \%$ reduction of the response time compared with the existing approaches. In our future work we plan to investigate the impact of the file size on the performance of the multi-source routing. We also plan to extend our proposed routing mechanism to support in-network content caching.

\section{REFERENCES}

[1] A. Feldmann, "Internet clean-slate design: What and why?" ACM SIGCOMM Computer Commun. Review, vol. 37, July 2017, pp. 59-64.

[2] M. Handley, "Why the Internet only just works," BT Technology Journal, vol. 24, no. 3, July 2006, pp. 119-129.

[3] T. Koponen, et al., "A data-oriented (and beyond) network architecture," Proc. ACM SIGCOMM'07, Kyoto, Japan, Aug. 2007. 
[4] V. Jacobson, et al., "Networking named content," Proc. 5th ACM International Conference on emerging Networking EXperiments and Technologies (CoNEXT), Rome, Italy, Dec. 2009.

[5] M. Brunner, "Scalable \& adaptive Internet solutions (SAIL)," Future Internet Assembly (FIA), Ghent, Belgium, Dec. 2010.

[6] G. Pavlou, N. Wang, W. K. Chai, and I. Psaras, "Internet-scale content mediation in information-centric networks," Annals of Telecommunications, Special Issue on Networked Digital Media, vol. 68, no. 3-4, April 2013, pp. 167-177.

[7] N. Fotiou, G. C. Polyzos, P. Nikander, and D. Trossen, "Developing information networking further: from PSIRP to PURSUIT," Proc. 7th International ICST Conference on Broadband Communications, Networks and Systems (BROADNETS), Athens, Greece, Oct. 2010.

[8] G. Xylomenos, C. N. Ververidis, V. A. Siris, N. Fotiou, C. Tsilopoulos, X. Vasilakos, K. V. Katsaros, and G. C. Polyzos, "A survey of information-centric networking research," IEEE Communications Surveys \& Tutorials, no. 99, July 2013, pp. 1-26.

[9] J. Choi, J. Han, E. Cho, T. Kwon, and Y. Choi, "Survey on content oriented networking for efficient content delivery," IEEE Communications Magazine, vol. 49, no. 3, March 2011, pp. 121-127.

[10] B. Ahlgren, C. Dannewitz, C. Imbrenda, D. Kutscher, and B. Ohlman, "A survey of information-centric networking," IEEE Communications Magazine, vol. 50, no. 7, July 2012, pp. 26-36.

[11] L. Mamatas, A. Papadopoulou, and V. Tsaoussidis, "Exploiting communication opportunities in disrupted network environments," Proc. 13rd International Conference on Wired/Wireless Internet Communications (WWIC), Malaga, Spain, May 2015.

[12] L. Wang. S. Bayhan, J. Ott, J. Kangasharju, A. Sathiaseelan, and J. Crowcroft, "Pro-diluvian: Understanding scoped-flooding for content discovery in information-centric networking," Proc. 2nd ACM Conference on Information-Centric Networking (ICN), San Francisco, USA, Sept. 2015.

[13] V. G. Vassilakis, L. Wang, L. Carrea, I. D. Moscholios, and M. D. Logothetis, "Scalable Bloom-filter based content dissemination in community networking using information centric principles," Proc. IEICE Information and Communication Technology Forum (ICTF), Patras, Greece, July 2016.

[14] G. Zhang, Y. Li, T. Lin, "Caching in information centric networking: A survey,” Computer Networks, vol. 57, Nov. 2013, pp. 3128-3141.

[15] V. G. Vassilakis, M. F. Al-Naday, M. J. Reed, B. A. Alzahrani, K. Yang, I. D. Moscholios, and M. D. Logothetis, "A cache-aware routing scheme for information-centric networks," Proc. 9th IEEE/IET International Symposium on Communication Systems, Networks \& Digital Signal Processing (CSNDSP), pp. 721-726, Manchester, U.K., July 2014.

[16] J. J. Garcia-Luna-Aceves, "Efficient multi-source multicasting in information centric networks," Proc. 12th IEEE Consumer Communications and Networking Conference (CCNC) - Industrial Program, Las Vegas, NV, USA, Jan. 9-12, 2015.

[17] L. Carrea, A. Vernitski, and M. J. Reed, "Optimized hash for network path encoding with minimized false positives," Computer Networks, vol. 58, January 2014, pp. 180-191.

[18] M. J. Reed, "Traffic engineering for information-centric networks," Proc. IEEE International Conference on Communications (ICC), Ottawa, Canada, June 2012

[19] J. Llorca, A. M. Turino, K. Guan, and D. Kilper, "Network-coded caching-aided multicast for efficient content delivery," Proc. IEEE International Conference on Communications (ICC), Budapest, Hungary, June 2013.

[20] Y. Liu and S.-Z. Yu, "Network coding-based multisource content delivery in content centric networking," Journal of Network and Computer Applications, vol. 64, April 2016, pp. 167-175.

[21] V. A. Siris, C. N. Ververidis, G. C. Polyzos, and K. P. Liolis, "Information centric networking (ICN) architectures for integration of satellites into the future Internet," Proc. 1st IEEE European Conference on Satellite Telecommunications (ESTEL), Rome, Italy, Oct. 2012.

[22] S. D'Oro, L. Galluccio, G. Morabito, and S. Palazzo, "SatCache: A profile-aware caching strategy for information-centric satellite networks," Transactions on Emerging Telecommunications Technologies, Wiley Online Library, vol. 25, no. 4, April 2014, pp. 436-444.
[23] B. A. Alzahrani, V. G. Vassilakis, and M. J. Reed, "Securing the forwarding plane in information centric networks," Proc. 5th Computer Science and Electronic Engineering Conference (CEEC), Colchester, U.K., Sept. 2013.

[24] B. A. Alzahrani, V. G. Vassilakis, and M. J. Reed, "Key management in information centric networking," International Journal of Computer Networks \& Communications, vol. 5, no. 6, Nov. 2013, pp. 153-166.

[25] X. Vasilakos, V. A. Siris, G. C. Polyzos, and M. Pomonis, "Proactive selective neighbor caching for enhancing mobility support in informationcentric networks," Proc. 2nd ACM Workshop on Information-Centric Networking (ICN), Helsinki, Finland, August 2012.

[26] M. F. Bari, S. R. Chowdhury, R. Ahmed, R. Boutaba, and B. Mathieu, "A Survey of naming and routing in information-centric networks," IEEE Communications Magazine, vol. 50, no. 12, Dec. 2012, pp. 44-53.

[27] B. Gajic, J. Riihijärvi, and P. Mähönen, "Intra-domain topology manager for publish-subscribe networks," Proc. 18th International Conference on Telecommunications (ICT), Traverse City, USA, July 2011.

[28] M. Särelä, C. E. Rothenberg, T. Aura, A. Zahemszky, P. Nikander, and J. Ott. "Forwarding anomalies in Bloom filter-based multicast," Proc. IEEE INFOCOM, Shanghai, China, April 2011.

[29] B. A. Alzahrani, V. G. Vassilakis, and M. J. Reed, "Mitigating bruteforce attacks on Bloom-filter based forwarding," Proc. Conference on Future Internet Communications (CFIC), Coimbra, Protugal, May 2013.

[30] S. Sengupta, M. Chen, P.A. Chou, and J. Li, "On optimality of routing for multi-source multicast communication scenarios with node uplink constraints," Proc. IEEE International Symposium on Information Theory (ISIT), Toronto, Canada, July 2008

[31] P. Ciciriello, L. Mottola, and G.P. Picco, "Efficient routing from multiple sources to multiple sinks in wireless sensor networks," Proc. 4th European Conference on Wireless Sensor Networks (EWSN), Delft, The Netherlands, January 2007.

[32] J. Zhang and Q. Zhang, "Cooperative routing in multi-source multidestination multi-hop wireless networks," Proc. IEEE INFOCOM, Phoenix, AZ, USA, April 2008.

[33] K. M. Mahmudul-Hoque, S. O. Amin, A. Alyyan, B. Zhang, L. Zhang, and L. Wang, "NLSR: Named-data link state routing protocol," Proc. 3rd ACM Workshop on Information Centric Networking (ICN), Hong Kong, August 2013, pp. 15-20.

[34] J. J. Garcia-Luna-Aceves, "Name-based content routing in information centric networks using distance information," Proc. 1st ACM International Conference on Information Centric Networking (ICN), Paris, France, Sept. 2014.

[35] M. Baugher, B. Davie, A. Narayanan, and D. Oran, "Self-verifying names for read-only named data," Proc. IEEE INFOCOM Workshops, Orlando, Florida, USA, March 2012.

[36] G. Kamel, N. Wang, V. Vassilakis, Z. Sun, P. Navaratnam, C. Wang, L. Dong, and R. Tafazolli, "CAINE: A context-aware information-centric network ecosystem," IEEE Communications Magazine, vol. 53, no. 8, August 2015, pp. 176-183.

[37] D. Rossi and G. Rossini, "On sizing CCN content stores by exploiting topological information," Proc. IEEE INFOCOM Workshop on NOMEN, Orlando, USA, March 2012.

[38] B. A. Alzahrani, M. J. Reed, J. Riihijärvi, and V. G. Vassilakis, "Scalability of information centric networking using mediated topology management," Journal of Network and Computer Applications, vol. 50, April 2015, pp. 126-133.

[39] N. Fotiou, G. Marias, and G. Polyzos, "Towards a secure rendezvous network for future publish/subscribe architectures," Proc. Future Internet Symposium (FIS), Berlin, Germany, September 2010.

[40] S. M. Bellovin, "Security problems in the TCP/IP protocol suite," ACM SIGCOMM Computer Communications Review, vol. 19, no. 2, April 1989, pp. 32-48.

[41] R. H. Khokhar, M. A. Ngadi, and S. Mandala, "A review of current routing attacks in mobile ad hoc networks," International Journal of Computer Science and Security, vol. 2, no. 3, 2008, pp. 18-29.

[42] The NS-3 network simulator. http://www.nsnam.org/ [May 2016]. 